\title{
Toward optical guidance during endoscopic ultrasound-guided fine needle aspirations of pancreatic masses using single fiber reflectance spectroscopy: a feasibility study
}

Paulien L. Stegehuis

Leonora S. F. Boogerd

Akin Inderson

Roeland A. Veenendaal

P. van Gerven

Bert A. Bonsing

J. Sven Mieog

Arjen Amelink

Maud Veselic

Hans Morreau

Cornelis J. H. van de Velde

Boudewijn P. F. Lelieveldt

Jouke Dijkstra

Dominic J. Robinson

Alexander L. Vahrmeijer

Paulien L. Stegehuis, Leonora S. F. Boogerd, Akin Inderson, Roeland A. Veenendaal, P. van Gerven, Bert A. Bonsing, J. Sven Mieog, Arjen Amelink, Maud Veselic, Hans Morreau, Cornelis J. H. van de Velde, Boudewijn P. F. Lelieveldt, Jouke Dijkstra, Dominic J. Robinson, Alexander L. Vahrmeijer, "Toward optical

SPIE. $\begin{aligned} & \text { guidance during endoscopic ultrasound-guided fine needle aspirations of pancreatic masses using } \\ & \text { single fiber reflectance spectroscopy: a feasibility study," J. Biomed. Opt. 22(2), } 024001 \text { (2017), }\end{aligned}$

SPIE. $\quad \begin{aligned} & \text { guidance during endoscopic ultrasound-guided fine needle aspirations of pancreatic masses using } \\ & \text { single fiber reflectance spectroscopy: a feasibility study," J. Biomed. Opt. 22(2), 024001 (2017), }\end{aligned}$ doi: $10.1117 / 1 . J B O .22 .2 .024001$. 


\title{
Toward optical guidance during endoscopic ultrasound-guided fine needle aspirations of pancreatic masses using single fiber reflectance spectroscopy: a feasibility study
}

\author{
Paulien L. Stegehuis, ${ }^{a, b, t}$ Leonora S. F. Boogerd,,${ }^{a, \dagger}$ Akin Inderson, ${ }^{c}$ Roeland A. Veenendaal, ${ }^{c}$ P. van Gerven, ${ }^{a}$ \\ Bert A. Bonsing, ${ }^{a}$ J. Sven Mieog, ${ }^{a}$ Arjen Amelink, ${ }^{\text {d }}$ Maud Veselic, ${ }^{e}$ Hans Morreau, ${ }^{e}$ Cornelis J. H. van de Velde, ${ }^{a}$ \\ Boudewijn P. F. Lelieveldt, ${ }^{b}$ Jouke Dijkstra, ${ }^{b}$ Dominic J. Robinson, ${ }^{f}$ and Alexander L. Vahrmeijer ${ }^{a, *}$ \\ aLeiden University Medical Center, Department of Surgery, Leiden, The Netherlands \\ 'Leiden University Medical Center, Department of Radiology, Leiden, The Netherlands \\ 'Leiden University Medical Center, Department of Gastroenterology and Hepatology, Leiden, The Netherlands \\ ${ }^{\mathrm{d}}$ Netherlands Organisation for Applied Scientific Research TNO, Department of Optics, Delft, The Netherlands \\ eLeiden University Medical Center, Department of Pathology, Leiden, The Netherlands \\ ${ }^{f}$ Center for Optical Diagnostics and Therapy, Department of Otolaryngology and Head and Neck Surgery, Erasmus MC, Rotterdam, \\ The Netherlands
}

\begin{abstract}
Endoscopic ultrasound-guided fine needle aspirations (EUS-FNA) of pancreatic masses suffer from sample errors and low-negative predictive values. Fiber-optic spectroscopy in the visible to near-infrared wavelength spectrum can noninvasively extract physiological parameters from tissue and has the potential to guide the sampling process and reduce sample errors. We assessed the feasibility of single fiber (SF) reflectance spectroscopy measurements during EUS-FNA of pancreatic masses and its ability to distinguish benign from malignant pancreatic tissue. A single optical fiber was placed inside a 19-gauge biopsy needle during EUS-FNA and at least three reflectance measurements were taken prior to FNA. Spectroscopy measurements did not cause any related adverse events and prolonged procedure time with $\sim 5 \mathrm{~min}$. An accurate correlation between spectroscopy measurements and cytology could be made in nine patients (three benign and six malignant). The oxygen saturation and bilirubin concentration were significantly higher in benign tissue compared with malignant tissue $(55 \%$ versus $21 \%, p=0.038 ; 166 \mu \mathrm{mol} / \mathrm{L}$ versus $17 \mu \mathrm{mol} / \mathrm{L}, p=0.039$, respectively). To conclude, incorporation of SF spectroscopy during EUS-FNA was feasible, safe, and relatively quick to perform. The optical properties of benign and malignant pancreatic tissue are different, implying that SF spectroscopy can potentially guide the FNA sampling. @ 2017 Society of Photo-Optical Instrumentation Engineers (SPIE) [DOI: 10.1117/1.JBO.22.2.024001]
\end{abstract}

Keywords: endoscopic-ultrasound-guided fine-needle aspiration; pancreas; single fiber; reflectance spectroscopy.

Paper 160791SSR received Nov. 16, 2016; accepted for publication Jan. 12, 2017; published online Feb. 6, 2017.

\section{Introduction}

Endoscopic ultrasound-guided fine needle aspiration (EUSFNA) is increasingly used as a diagnostic modality to obtain a cytological diagnosis of suspect pancreatic masses. Surgery is currently the only treatment option for long-term survival in patients with pancreatic cancer. However, only a small minority of patients is eligible for surgery due to late detection, and it is often performed without pathological confirmation of the diagnosis. ${ }^{1}$ Neoadjuvant therapy gains more interest to downsize the primary tumor and treat micrometastatic disease prior to resection. ${ }^{2}$ Consequently, the need for a pathological confirmation is warranted before the start of chemotherapy. ${ }^{3,4}$ Although EUS-FNA is a relatively safe procedure with a high specificity, it suffers from a high sampling error, resulting in a low-negative predictive value, and the need for an on-site cytopathologist. ${ }^{5,6}$ Moreover, the accuracy of EUS-FNA drops considerably when (peritumoral) pancreatitis is present, which most frequently occurs. ${ }^{7}$

\footnotetext{
${ }^{*}$ Address all correspondence to: Alexander L. Vahrmeijer, E-mail: a.I. vahrmeijer@lumc.nl
}

†Both authors contributed equally to the study and share first authorship.
Several techniques have been investigated to enhance the diagnostic accuracy including contrast enhancement, confocal laser endomicroscopy, and optical coherence tomography. ${ }^{8-12}$ However, these techniques involve administration of a contrast agent or extensive training to interpret the images. Fiber-optic spectroscopy is a relatively novel diagnostic modality that already showed its feasibility in a pilot study to characterize pancreatic tissue and to differentiate between normal and malignant tissue during surgery. ${ }^{13}$ Although these first in vivo results were encouraging, the size of the probe was not compatible with the small lumen of an FNA needle. No studies have yet been performed to assess the feasibility of fiber-optic spectroscopy during pancreatic EUS-FNA procedures.

Reflectance spectroscopy provides information about tissue absorption and scattering parameters. Light in the visible to near-infrared wavelength range is dominantly absorbed in the biological tissue by oxygenated and deoxygenated hemoglobin. Light is also absorbed by other chromophores, such as bilirubin and beta-carotene, depending on the tissue type. Single fiber (SF) reflectance spectroscopy uses only an SF to both emit

$1083-3668 / 2017 / \$ 25.00$ @ 2017 SPIE 
the outgoing and capture the returning light. The advantage of using an SF is its ability to fit through an endoscopic biopsy needle, which, combined with its small sampling depth of approximately the fiber diameter, ${ }^{14}$ allows measurements at the exact biopsy location. Using a priori knowledge of the absorption spectra of the chromophores, a previously developed, described, and validated mathematical model can be used to extract and quantify physiological information, such as tissue oxygenation and blood volume fraction, from the obtained spectra. ${ }^{14,15}$ In previous studies, our group has shown the potential of SF reflectance spectroscopy in detecting abnormal mediastinal lymph nodes during EUS-FNA procedures in patients with a clinical suspicion of lung cancer. ${ }^{16}$

In this study, we evaluated the feasibility of the incorporation of SF reflectance spectroscopy into pancreatic EUS-FNA procedures. The primary objectives were to assess the safety-assessed through adverse events - and ease of use - assessed through additional time taken-during the procedure. Moreover, we measured wavelength-dependent optical characteristics of pancreatic masses and assessed whether we could distinguish benign from malignant pancreatic tissue based on their optical properties.

\section{Material and Methods}

\subsection{Patients}

This trial was approved by the Medical Ethics Committee of the Leiden University Medical Center and was performed in accordance with the ethical standards of the Helsinki Declaration of 1975. Sixteen patients planned to undergo an EUS-FNA procedure to obtain a diagnosis of a pancreatic mass were included. All patients provided informed consent and were anonymized. The EUS-FNA procedures were performed by two experienced gastroenterologists.

\subsection{Measurement Setup}

The experimental setup used in this study has been described in detail previously ${ }^{15}$ and is shown in Fig. 1. In short, the setup utilizes a single optical fiber connected to a quadfurcated optical fiber. Two arms are connected; one arm leading from a halogen light source HL-2000-FHSA (Ocean Optics, the Netherlands), and a second arm leading to a spectrophotometer SD-2000 (Ocean Optics, the Netherlands) to measure white light reflectance. A calibration procedure was performed to account for internal reflections, variability in lamp-specific output and in fiber-specific transmission properties. ${ }^{17}$

Sterilized single-use fibers (Leoni, Germany) had a core diameter of $300 \mu \mathrm{m}$, an outer diameter of $700 \mu \mathrm{m}$, an SMA905 connector, a distal polished fiber for wide-angle beam, a numerical aperture of 0.22 , and a length of $3 \mathrm{~m}( \pm 0.2 \mathrm{~m})$. The sampling depth depends on the optical properties but is typically similar to the core diameter of the fiber. ${ }^{14}$

\subsection{Examination Procedure}

The procedure was performed using a curved linear array echo endoscope EG-327UK Slim, EG-3870UTK (Pentax Europe GmbH, Hamburg, Germany) or EG-580UT (Fujifilm, Tokyo, Japan). Patients were positioned in left lateral decubitus position under conscious sedation ( $50 \mathrm{mcg}$ fentanyl, $3 \mathrm{mg}$ midazolam) or deep sedation (propofol, administered by nurse anesthesist). After visualization of the pancreatic mass, the stylet of a 19-G biopsy needle (ECHO-19; Cook Medical, Bloomington) was replaced by a sterilized single-use fiber, after which the needle was placed into the endoscope channel and advanced into the target lesion. The fiber was extended a few millimeters through the biopsy needle to enable measurements without influence of the needle shaft. At least three reflectance measurements were acquired before FNA. At the time of these measurements, systemic blood saturation was measured using a pulse oximeter. Individual reflectance spectroscopy measurements were averaged over 10 measurements with an integration time of $100 \mathrm{~ms}$. After obtaining spectroscopy measurements, the fiber was removed and the routine procedure was continued. The cytology slides with the resulting FNA were marked to correlate spectroscopy measurements to cytology outcome. The puncture was repeated a maximum of 5 times until the on-site cytopathologist had confirmed the adequacy of the sample. Spectroscopy measurements were only taken prior to the first puncture.

\subsection{Pathologic Assessment of Cytology}

The aspirated material was examined on-site to judge the sample for its adequacy. Punction material was expelled from the needle onto the glass slides and gently smeared. Air-dried smears were stained using the rapid RAL staining (RAL Diagnostics, Martical, France). Remaining material was formalin fixed; this material gives opportunity of performing ancillary
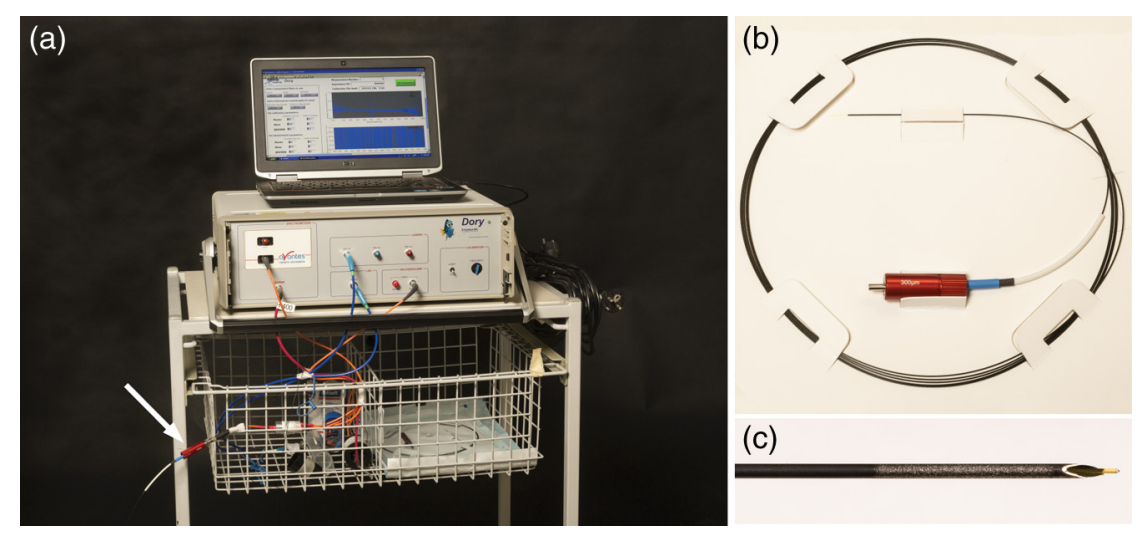

Fig. 1 Measurement setup. (a) The measurement device with laptop. The white arrow points at the connection point for the fiber. (b) The optical fiber, with a length of $3 \mathrm{~m}$ and an outer diameter of $700 \mu \mathrm{m}$. (c) Incorporation of the optical fiber into 19-G puncture needle. 
techniques. After the procedure, all slides were examined by an experienced cytopathologist.

\subsection{Mathematical Analysis of Spectra}

SF reflectance spectra were analyzed using an analytical model to describe the wavelength-dependent optical properties to extract physiological and morphological information from the sampled tissue. Previously, a similar model has been described for analysis of other tissues. ${ }^{18}$ Attenuation due to absorption within the tissue is modeled using a modified Beer-Lambert law and is a function of both the tissue absorption coefficient $\left(\mu_{a}\right)$ and the SF photon path length. The reflectance amplitude, as well as the SF photon path length, depend on the scattering properties of the tissue, with a dependence on the reduced scattering coefficient $\left(\mu_{s}^{\prime}\right)$ and on the angular distribution of scattering (phase function), modeled through phase function parameter $\gamma=\left(1-g_{2}\right) /\left(1-g_{1}\right)$ with $g_{1}$ and $g_{2}$ the first and second moments of the phase function, respectively. The dependence of the SF reflectance signal on phase function parameter gamma results from the overlapping source-detector areas utilized in SF measurements, for which the diffusion approximation does not hold. ${ }^{19}$ The complete model used to fit the data is given in the following equation:

$$
\begin{aligned}
& R_{\mathrm{SF}}^{\mathrm{Model}}\left(\mu_{a}, \mu_{s}^{\prime}, \gamma, d_{\mathrm{fib}}, \mathrm{NA}, \eta_{\text {medium }}\right) \\
& =\frac{\mathrm{NA}^{2}}{\eta_{\text {medium }}^{2}}\left[1+0.62 \gamma^{2} e^{-2.3 \gamma^{2}\left(\mu_{s}^{\prime} d_{\mathrm{fib}}\right)}\right]\left[\frac{\left(\mu_{s}^{\prime} d_{\mathrm{fib}}\right)^{0.57 \gamma}}{2.3 \gamma^{2}+\left(\mu_{s}^{\prime} d_{\mathrm{fib}}\right)^{0.57 \gamma}}\right] \\
& \times e^{-\mu_{a} \frac{1.05 \gamma^{0.6} d_{\mathrm{fib}}}{\left(\mu_{s}^{\prime} d_{\mathrm{fib}}\right)^{0.18}\left[0.64+\left(\mu_{a} d_{\mathrm{fib}}\right)^{0.64]}\right.},}
\end{aligned}
$$

where $d_{\mathrm{fib}}$ is the fiber core diameter and NA is the numerical aperture of the fiber $(0.3 \mathrm{~mm}$ and 0.22 , respectively $)$ and $\eta_{\text {medium }}$ is the refractive index of the pancreatic tissue under investigation, assumed to be $1.38 .^{20}$ The reduced scattering coefficient was fitted as a power-law function, ${ }^{17} \mu_{s}^{\prime}=a_{0}\left(\lambda / \lambda_{0}\right)^{a 1}$. Gamma was assumed to be constant over the fitted wavelength range, $\gamma=a_{2}$. Furthermore, instead of fitting $\gamma$ as a free parameter, we have also fitted Eq. (1) keeping $\gamma$ fixed at 1.4, 1.6, and 1.8 , which is an expected range for $\gamma$ in biological tissues. ${ }^{21} \mathrm{We}$ have verified that these different approaches for fitting the data to Eq. (1) did not result in differences in the estimated absorption coefficient of more than $6 \%$.

Since we have not previously performed measurements on pancreatic tissue, our initial model assumed that absorption was attributable to oxygenated $\left(\mathrm{HbO}_{2}\right)$ and deoxygenated hemoglobin $(\mathrm{Hb})$ confined within the local microvasculature and bilirubin according to the following equation:

$\mu_{a}^{\mathrm{tissue}}=a_{3} C_{v}\left[a_{4} \mu_{a}^{\mathrm{HbO}_{2}}+\left(1-a_{4}\right) \mu_{a}^{\mathrm{Hb}}\right]+\mu_{a}^{\mathrm{BIL}} a_{5}$,

where $a_{3}$ is the blood volume fraction, $a_{4}$ is the microvascular hemoglobin oxygen saturation, $C_{\mathrm{v}}$ is a factor that accounts for the effect of discrete blood vessels on the absorption coefficient and enables an estimation of the average blood vessel diameter $d_{\mathrm{v}}=a_{6}, a_{5}$ is the bilirubin concentration, $\mu_{a}^{\mathrm{BIL}}$ is the specific absorption coefficient of bilirubin, and $\mu_{a}^{\mathrm{HbO}_{2}}$ and $\mu_{a}^{\mathrm{Hb}}$ are the specific absorption coefficients of oxy- and deoxyhemoglobin, respectively.

A Levenberg-Marquardt algorithm was used to estimate the parameter values $a_{1}-a_{6}$ by minimizing the chi-squared metric between measured reflectance data and model predictions.
Confidence intervals on parameter estimates were calculated from the square root of the diagonal of the covariance matrix. ${ }^{22}$ Parameter values were averaged over repeated measurements, weighted by the confidence interval of individual spectral fits, and reported with the associated weighted standard deviation.

In this study, spectra that showed evidence of a blood pool within the detection volume were identified by blood volume fraction $a_{3}>40 \%$ and excluded from the analysis as was done previously. ${ }^{18}$

\subsection{Statistical Analysis}

The statistical analysis and the graphs were generated using SPSS statistical software (Version 22.0, Chicago, Illinois). Statistical analysis was carried out using the Kruskal-Wallis test on the parameters obtained from the fit. This test replaces data by rank and is considered adequate when data does not show a Gaussian distribution. ${ }^{23}$

\section{Results}

\subsection{Patients}

Sixteen patients undergoing a pancreatic EUS-guided FNA were included. In three patients no FNA, and thus no spectroscopic measurements, was performed, due to lack of visualization of the pancreatic mass. In the remaining thirteen patients, we were able to perform spectroscopic measurements. Of these, two patients were excluded due to a poor connection between the sterile measurement fiber and the quadfurcated optical fiber causing erroneous spectra and two patients were excluded due to lack of precise correlation with pathology (Fig. 2). In the latter two patients, the yield of the 19-G needle biopsies was inadequate and instead, good quality FNAs were acquired with the more flexible and smaller 25-G needle. One of the excluded patients was a patient with a pancreatic neuroendocrine tumor; the final puncture, obtained with a $25-\mathrm{G}$ needle, was performed at the same location where the 19-G needle was positioned and spectroscopy measurements were taken. Because the cytology correlation might not be completely reliable, we described SF

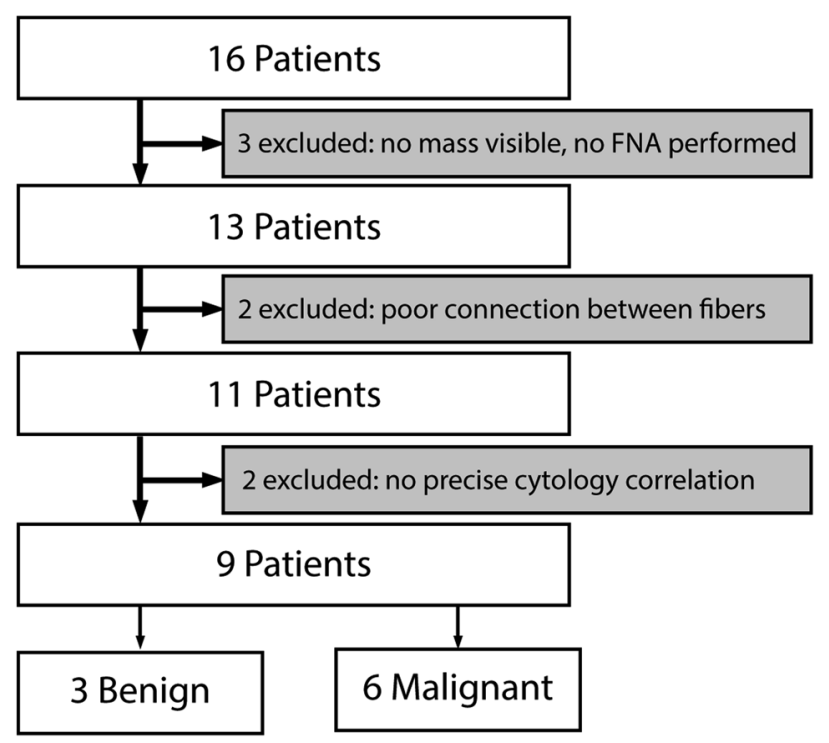

Fig. 2 Inclusion scheme of patients. 
Table 1 Patient characteristics. Patients 3, 6, and 12 were included, but no biopsy was taken so no SF reflectance spectroscopy measurements were performed.

\begin{tabular}{|c|c|c|c|c|c|}
\hline Patient & Gender & Age & Site & Diameter tumor $(\mathrm{mm})$ & Cytology \\
\hline 1 & M & 71 & Corpus & 30 & Malignant: mucinous cystic tumor \\
\hline 2 & M & 74 & Corpus/tail & 80 & Malignant: adenocarcinoma \\
\hline $4^{\mathrm{a}}$ & $\mathrm{V}$ & 47 & Head & NS & Neuroendocrine tumor \\
\hline 5 & $\mathrm{~F}$ & 54 & Head & NS & Malignant: metastasis adenocarcinoma ovary \\
\hline 7 & $\mathrm{~F}$ & 70 & Whole pancreas & 50 & Benign: fibrotic inflammation \\
\hline $8^{\mathrm{a}}$ & M & 47 & Head & 25 & Malignant: highly dysplastic cells \\
\hline $9^{a}$ & M & 63 & Head and tail & NS & Benign: autoimmune pancreatitis \\
\hline 10 & $\mathrm{~F}$ & 55 & Tail & 40 & Malignant: adenocarcinoma \\
\hline 11 & M & 60 & Corpus & 33 & Benign: autoimmune pancreatitis \\
\hline 13 & $\mathrm{~F}$ & 60 & Processus Uncinatus & NS & Malignant: adenocarcinoma \\
\hline 14 & $\mathrm{~F}$ & 53 & Processus Uncinatus & 24 & Malignant: adenocarcinoma \\
\hline $15^{\mathrm{a}}$ & M & 69 & Head/Processus Uncinatus & 44 & Benign: fibrosis \\
\hline 16 & $\mathrm{M}$ & 74 & Head & 20 & Benign: fibrosis \\
\hline
\end{tabular}

Note: NS, not specified.

${ }^{\mathrm{a} S p e c t r o s c o p y ~ m e a s u r e m e n t s ~ o f ~ t h e s e ~ p a t i e n t s ~ w e r e ~ n o t ~ t a k e n ~ i n t o ~ a c c o u n t ~ f o r ~ t h e ~ f i n a l ~ a n a l y s i s . ~}$

reflectance spectra of this patient separately. Characteristics of these patients are listed in Table 1.

Cytological results could be correlated with spectroscopy measurements in the remaining nine patients, with cytological diagnosis of the spectroscopy-related FNA sample showing malignancy in six patients and benign disease in three patients. Two out of the three patients whose cytology showed a benign anomaly were at the end of their treatment plan suspect to suffer from pancreatic cancer. Patient \#7 diagnosed with fibrotic inflammation, underwent pancreatic surgery based on clinical symptoms and preoperative images. The surgery was aborted due to hemodynamic instability. Patient \#16, diagnosed with fibrosis, showed an increase in tumor volume and thickened lymph nodes on a CT-scan, performed one month after the EUS-FNA. Both patients died within four months after the EUS-FNA procedure. Although no final histopathological diagnosis could be made, this was most likely due to a pancreatic adenocarcinoma.

\subsection{Single Fiber Reflectance Spectra}

No adverse events related to the spectroscopy measurements were observed. Additional endoscopy time related to the spectroscopic measurements was approximately five minutes.

Representative SF reflectance spectra from measurements taken at a benign and malignant spot (patients \#16 and \#2) are displayed in Fig. 3. In Table 2, the measured values of blood oxygen saturation, blood volume, and bilirubin concentration are displayed. Pancreatic tissue does not show much hemoglobin absorption features in the spectra, which are typically visible as "dips" in reflectance between 500 and $600 \mathrm{~nm}$. The lack of strong absorption features in the spectra is reflected in the fitted values for the blood volume, which are low for both benign and malignant sites $(1.1 \%$ and $2.2 \%$, respectively; Fig. 4). The microvascular saturation is significantly higher at benign sites than malignant sites $(55 \%$ versus $21 \%$, $p=0.038$; Fig. 4). Bilirubin absorbs light mostly between 400 and $500 \mathrm{~nm}$, with a peak around $450 \mathrm{~nm}$. Benign sites were associated with significantly higher bilirubin content than malignant sites $(166 \mu \mathrm{mol} / \mathrm{L}$ versus $17 \mu \mathrm{mol} / \mathrm{L}$, $p=0.039$; Fig. 4). The amplitude and slope of the reduced scattering coefficient ( $a_{0}$ and $a_{1}$, respectively) were not significantly different between benign and malignant tissue. Measurements of the patient who suffered from a pancreatic neuroendocrine tumor showed a blood level of $33.6 \% \pm 1.6 \%$ with a saturation of $86.2 \% \pm 12.1 \%$.

\section{Discussion}

In this study, we showed that the incorporation of SF spectroscopic measurements during EUS-FNA procedures of the pancreas is feasible and safe. Because the fiber can be preloaded, measurements can be performed relatively easily and quickly. In our pilot study, the endoscopic procedure was prolonged with $\sim 5 \mathrm{~min}$ on average, which could be shortened after gaining more experience. No adverse events related to the spectroscopy measurements were observed, making it a safe procedure. Moreover, we demonstrated that optical properties, extracted from the SF reflectance spectra, appeared to be different between benign and malignant pancreatic aspirations. Therefore, incorporation of fiber-optic spectroscopy could potentially help to guide the FNA procedures and thereby reduce the sampling error and increase its negative predictive value without significantly affecting the complexity or time of the procedure. 

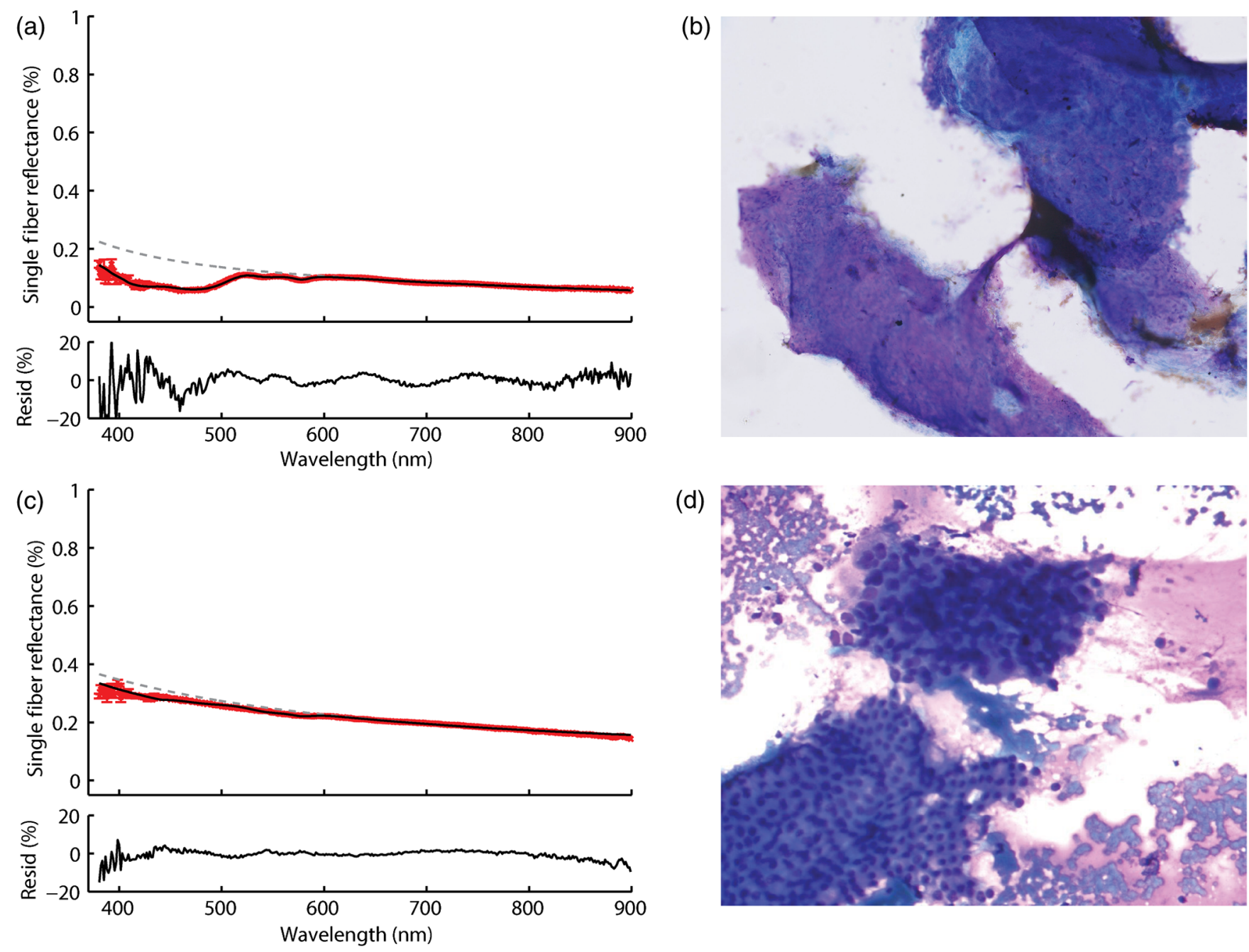

(d)

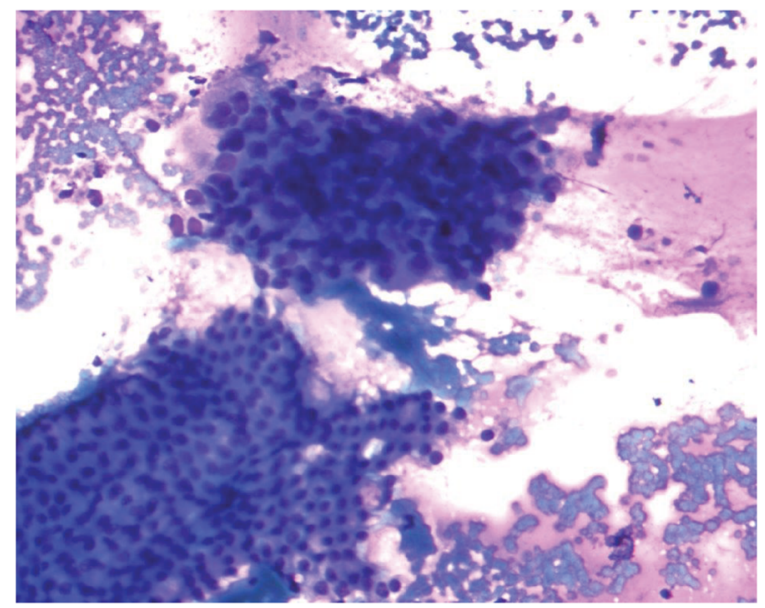

Fig. 3 Fitted spectra with corresponding cytology. (a) and (c) Spectra of a benign and malignant sample, respectively. The red line in the spectra indicates the actual measurements, and the black line shows the fit. The dotted line represents the expected spectra if no absorbers would be present in the sample. The residual-difference between measurement and fit-is shown below the spectra. (b) The corresponding cytology sample of (a): the lower left corner shows fibrosis (low magnification). (d) The corresponding cytology samples of (c): pancreatic ductal adenocarcinoma cells are shown in the middle (high magnification).

Although EUS-FNA currently has a high specificity, it suffers from a low-negative predictive value implying that a negative result cannot be relied upon. ${ }^{24}$ This is also confirmed by the current study: cytology results of three out of the nine patients showed a benign condition. Two out of these three patients (fibrotic inflammation and fibrosis), eventually turned out to have a malignancy by follow-up treatment or diagnosis and the FNA results thus represent sample errors. As more attention is given to neoadjuvant chemotherapy, ${ }^{2}$ which requires a pathological diagnosis, it is highly important to reduce the sampling error and increase the negative predictive value of EUSFNA procedures.

Pancreatic ductal adenocarcinoma is a hypovascular and a hypoxic tumor. ${ }^{25-27}$ This is confirmed in this study, since both the benign and malignant sites showed very low levels of mean blood volume (1.1\% and $2.1 \%$, respectively). These results are supported by a study of Erkan et al., ${ }^{25}$ in which the microvascular density was determined: normal pancreas showed a five times higher microvascular density than fibrotic areas of chronic pancreatitis, and four times higher than pancreatic ductal adenocarcinoma. Interestingly, one of the patients in which we took measurements (but lacked good cytological correlation) was diagnosed with a pancreatic neuroendocrine tumor, which are known to be hypervascular. ${ }^{28}$ That measurement indeed showed a far higher blood level of $33.6 \% \pm 1.6 \%$ with a saturation of $86.2 \% \pm 12.1 \%$.

The saturation of hemoglobin is a marker for the level of hypoxia. ${ }^{29,30}$ We found significant lower mean saturation values in malignant areas compared to benign areas (21\% and 55\%, respectively), despite high overall arterial oxygen saturation values $(>97 \%)$. This is in agreement with results of Koong et al., ${ }^{31}$ who intraoperatively measured pancreatic tissue oxygenation (using an Eppendorf $\mathrm{pO}_{2}$ histograph) and found significant tumor hypoxia, whereas normal tissue showed normal oxygenation. In another study, patients undergoing pancreatic surgery received pimonidazole - a hypoxia tracer that can be detected in tissue by immunohistochemistry-and showed that more hypoxia was present in the epithelial regions than in the stromal 
Table 2 Extracted means and standard deviations of blood volume, saturation, and bilirubin per patient.

\begin{tabular}{|c|c|c|c|c|c|c|c|}
\hline \multirow[b]{2}{*}{ Patient } & \multirow[b]{2}{*}{ Cytology result } & \multicolumn{2}{|c|}{ Blood volume (\%) } & \multicolumn{2}{|c|}{ Saturation (\%) } & \multicolumn{2}{|c|}{ Bilirubin $(\mu \mathrm{mol} / \mathrm{L})$} \\
\hline & & Mean & SD & Mean & SD & Mean & SD \\
\hline 1 & Malignant & 0.7 & 0.3 & 29.4 & 45.9 & 2.5 & 1.8 \\
\hline 2 & Malignant & 0.9 & 0.5 & 20.9 & 5.0 & 7.2 & 2.7 \\
\hline 5 & Malignant & 1.4 & 0.4 & 0.0 & 7.8 & 11.8 & 3.1 \\
\hline 7 & Benign & 0.3 & 0.1 & 48.3 & 30.3 & 39.2 & 18.1 \\
\hline 10 & Malignant & 5.1 & 4.1 & 0.0 & 50.0 & 3.4 & 2.4 \\
\hline 11 & Benign & 0.8 & 0.3 & 50.8 & 42.4 & 338.8 & 239.7 \\
\hline 13 & Malignant & 3.1 & 1.9 & 49.6 & 4.2 & 26.6 & 0.5 \\
\hline 14 & Malignant & 1.8 & 0.2 & 28.7 & 8.9 & 48.6 & 3.2 \\
\hline 16 & Benign & 2.0 & 1.5 & 64.9 & 18.0 & 119.3 & 95.1 \\
\hline
\end{tabular}

Note: SD, standard deviation.
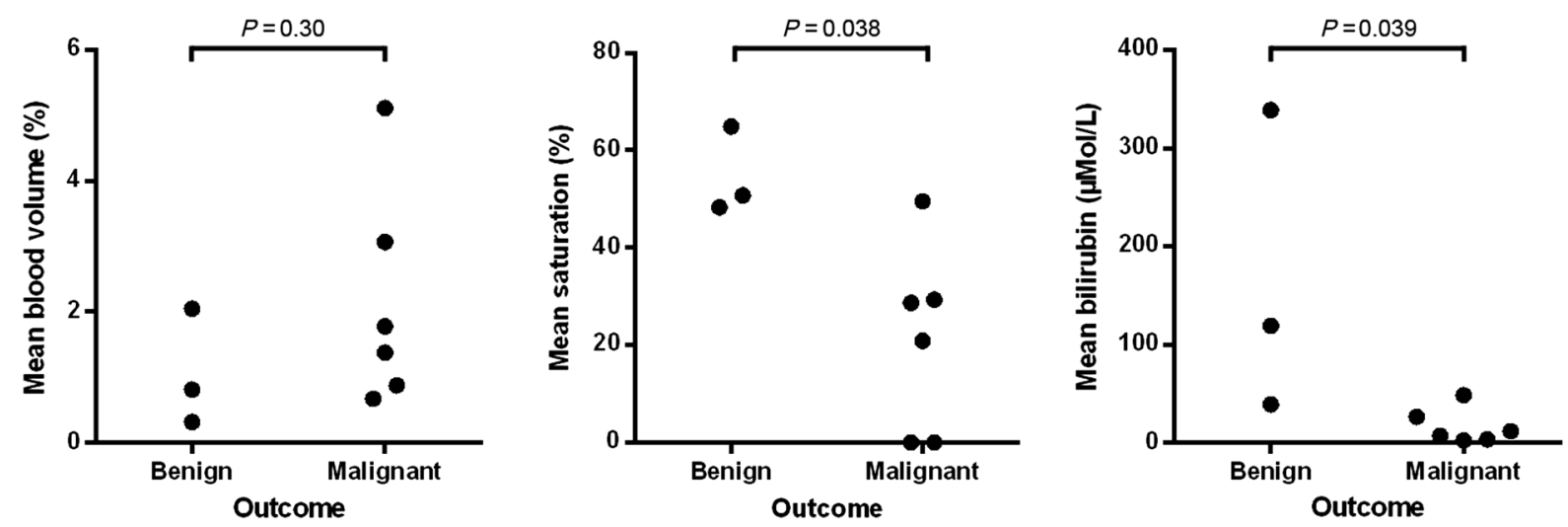

Fig. 4 Difference in blood volume, saturation, and bilirubin concentration between cytological confirmed benign and malignant sites. Every individual patient is indicated by a dot. $P$-values are displayed above the scatterplots.

regions of the tumor (although there was a high intra- and intertumoral heterogeneity) and the absence of hypoxia in adjacent normal pancreatic tissue. ${ }^{32}$ Additionally, Lohse et al. ${ }^{33}$ measured hypoxia in the stromal compartment of patient-derived pancreatic xenografts. They concluded that the levels of hypoxia in the tumor-associated stroma were generally lower when compared to the tumor compartment. These studies support our results. Although we did not measure normal tissue, we did-most likely-measure stromal areas of pancreatic adenocarcinoma in the two patients who suffered from fibrosis and inflammation (according to the cytology results obtained through EUS-FNA). These patients indeed showed a higher saturation and thus lower hypoxia levels than epithelial pancreatic adenocarcinoma regions. Furthermore, hypoxia is a known biomarker and potential therapeutic target in pancreatic cancer ${ }^{34-36}$ and in vivo saturation measurements could provide interesting additional information to increase our understanding of the role of hypoxia before and during neoadjuvant treatment.

The levels of bilirubin found in this study are within the range that could be expected for this patient cohort.
However, we cannot directly correlate the bilirubin concentration measured in pancreatic tissue to the bilirubin concentration measured in plasma, since we are only optically sampling a small amount of tissue with a low blood volume fraction. Moreover, we did not prospectively measure serum bilirubin levels prior to EUS-FNA. This would be a valuable addition as bilirubin levels can change quickly in cholestatic patients. It is interesting to speculate on the reason for our observation of lower bilirubin levels in malignant tissue compared to benign tissue. This may be a consequence of the (isolated) tumor microenvironment characterized by low blood volume, combined with the reduced availability of heme in rapidly proliferating tumor cells ${ }^{37}$ and the possible degradation of bilirubin, known to be a strong antioxidant ${ }^{38}$ in tumor cells under oxidative and (inflammatory) stress.

One other clinical study described in vivo spectroscopy measurements in human pancreatic tissue. ${ }^{13}$ It was concluded that a difference between normal and malignant tissue could be observed in collected reflectance at wavelengths around 470 and $650 \mathrm{~nm}$, measured with a multifiber probe. However, the 
diameter of the multifiber probe is too large to fit through an endoscopic biopsy needle, making a translation to standard EUS-FNA procedures difficult.

In this study, reflectance spectra were analyzed using a fit algorithm [Eq. (1)] that incorporates the fact that the reflectance amplitude and the SF photon path length depend on the scattering properties of the tissue, with a dependence on not just the reduced scattering coefficient $\left(\mu_{s}^{\prime}\right)$ but also on the angular distribution of scattering (phase function) modeled through phase function parameter $\gamma$. We note that the use of Eq. (1) to fit the data no longer requires the use of the method described in a study by Kanick et al. ${ }^{39}$ In that study, instead of explicitly expressing the $\gamma$ dependence of the collected SF reflectance, a set of empirical constants was used that minimized the error between the true and fitted SF path lengths. However, since the combined effects of $\mu_{a}, \mu_{s}^{\prime}$, and $\gamma$ on SF reflectance are now fully captured by Eq. (1), such an approach based on empirical constants is no longer necessary for accurate estimation of $\mu_{a}$. The fact that the different methods of fitting to Eq. (1) did not result in differences in the estimated $\mu_{a}$ of more than $6 \%$ indicates that this new method of fitting is robust.

A limitation of our study is the overall small sample size and small number of benign tissue measurements. However, our primary aim was to study the feasibility of incorporating SF reflectance spectroscopy measurements into the EUS-FNA procedure. Although it is encouraging that even with a small sample size differences in optical properties are observed, a larger (multicenter) study, including a wide variety of pancreatic lesions, is needed to fully exploit the potential of SF spectroscopy to guide the EUS-FNA sampling. In this study, we grouped pancreatitis with fibrous tumor parts (that were regarded as benign by the cytologist) together. More samples would enable us to also study the differences between these individual groups. The group of Hoffman et al., developed an orthotopic human tumor graft mouse model in which different fluorescent proteins were tested to image tumor progression. ${ }^{40,41}$ This approach could be interesting as it allows visual distinction between pancreatic cancer cells and the surrounding stroma. ${ }^{42,43}$ In addition, several clinical trials are currently investigating the safety and feasibility of intraoperative near-infrared fluorescence imaging using pancreatic cancer-specific contrast agents (NTR5673, NCT02736578).

The fiber used in this study fits through a 19-G needle, but is too thick for the more flexible 22-G needle, which is currently the preferred size at our gastroenterology department. Furthermore, we noticed that the SMA-connector of the sterile fiber was not always easily connected to the quadfurcated fiber, causing erroneous reflections in two patients. Future studies will, therefore, be performed with a smaller diameter fiber that fits through a 22-G needle, and with tighter tolerance requirements on the SMA-connector to secure a tight connection between fiber and measurement setup.

Real-time feedback is crucial to guide the gastroenterologist to epithelial tumor regions during the procedure, and, since our spectral fitting method is sufficiently fast $(<1 \mathrm{~s})$, this is feasible. Finally, to make the incorporation of SF reflectance spectroscopy into the FNA procedure even more simple, the small SF could potentially be integrated into the shaft of the biopsy needle, as already shown in a $15-\mathrm{G}$ needle. ${ }^{44}$ Integration of the optic fiber would allow direct visualization of the difference between benign and malignant tissue per patient, as we currently do not have data on intrapatient differences.
In conclusion, incorporation of SF reflectance spectroscopy measurements in EUS-FNA procedures of the pancreas is feasible and safe. Moreover, the optical properties differ between benign and malignant tissue sites, which could be used for realtime guidance of FNA sampling to reduce the sample error of these procedures and improve overall performance.

\section{Disclosures}

The authors declare no conflicts of interest, financial or otherwise.

\section{Acknowledgments}

Work at the LUMC was funded by the European Union Seventh Framework Program FP7-ICT-2011-8under Grant Agreement No. 318729 (CAReIOCA project), the Dutch Cancer Society under Grant Agreement No. UL2010-4732, and the Bas Mulder Award (Grant No. UL2015-7665) from the Alpe d'HuZes Foundation/Dutch Cancer Society.

\section{References}

1. E. P. Tamm et al., "Imaging of pancreatic adenocarcinoma: update on staging/resectability," Radiol. Clin. North Am. 50(3), 407-428 (2012).

2. S. Tsai and D. B. Evans, "Therapeutic advances in localized pancreatic cancer," J. Am. Med. Assoc. Surg. 74(11), 2913-2921 (2016).

3. J.-M. Dumonceau et al., "Indications, results, and clinical impact of endoscopic ultrasound (EUS)-guided sampling in gastroenterology: European Society of Gastrointestinal Endoscopy (ESGE) clinical guideline," Endoscopy 43(10), 897-912 (2011).

4. M. Hidalgo, "Pancreatic cancer," N. Engl. J. Med. 362(17), 1605-1617 (2010).

5. M. A. Eloubeidi et al., "Yield of endoscopic ultrasound-guided fine-needle aspiration biopsy in patients with suspected pancreatic carcinoma," Cancer 99(5), 285-292 (2003).

6. J. Chen et al., "Diagnostic accuracy of endoscopic ultrasound-guided fine-needle aspiration for solid pancreatic lesion: a systematic review," J. Cancer Res. Clin. Oncol. 138(9), 1433-1441 (2012).

7. A. Fritscher-Ravens et al., "Comparison of endoscopic ultrasoundguided fine needle aspiration for focal pancreatic lesions in patients with normal parenchyma and chronic pancreatitis," Am. J. Gastroenterol. 97(11), 2768-2775 (2002).

8. K. Kamata et al., "New techniques of endoscopic ultrasonography for the pancreaticobiliary diseases," Ultrasonography 35(3), 169-179 (2016).

9. K. Grieve et al., "A feasibility study of full-field optical coherence tomography for rapid evaluation of EUS-guided microbiopsy specimens," Gastrointest. Endosc. 81(2), 342-350 (2015).

10. V. J. A. Konda et al., "First assessment of needle-based confocal laser endomicroscopy during EUS-FNA procedures of the pancreas (with videos)," Gastrointest. Endosc. 74(5), 1049-1060 (2011).

11. R. Regunathan et al., "Feasibility and preliminary accuracy of high-resolution imaging of the liver and pancreas using FNA compatible microendoscopy (with video)," Gastrointest. Endosc. 76(2), 293-300 (2012).

12. N. K. Reddy et al., "Contrast-enhanced endoscopic ultrasonography," World J. Gastroenterol. 17(1), 42-48 (2011).

13. W. R. Lloyd et al., "In vivo optical spectroscopy for improved detection of pancreatic adenocarcinoma: a feasibility study," Biomed. Opt. Express 5(1), 9-15 (2014).

14. S. C. Kanick et al., "Monte Carlo analysis of single fiber reflectance spectroscopy: photon path length and sampling depth," Phys. Med. Biol. 54(22), 6991-7008 (2009).

15. S. C. Kanick, H. J. C. M. Sterenborg, and A. Amelink, "Empirical model of the photon path length for a single fiber reflectance spectroscopy device," Opt. Express 17(2), 860-871 (2009).

16. S. C. Kanick et al., "Characterization of mediastinal lymph node physiology in vivo by optical spectroscopy during endoscopic ultrasoundguided fine needle aspiration," J. Thorac. Oncol. 5(7), 981-987 (2010). 
17. F. van Leeuwen-van Zaane et al., "In vivo quantification of the scattering properties of tissue using multi-diameter single fiber reflectance spectroscopy," Biomed. Opt. Express 4(5), 696-708 (2013).

18. S. C. Kanick et al., "Integration of single-fiber reflectance spectroscopy into ultrasound-guided endoscopic lung cancer staging of mediastinal lymph nodes," J. Biomed. Opt. 15(1), 017004 (2010).

19. U. A. Gamm et al., "Quantification of the reduced scattering coefficient and phase-function-dependent parameter $\gamma$ of turbid media using multidiameter single fiber reflectance spectroscopy: experimental validation," Opt. Lett. 37(11), 1838-1840 (2012).

20. S. Y. Lee et al., "Characterizing human pancreatic cancer precursor using quantitative tissue optical spectroscopy," Biomed. Opt. Express 4(12), 2828 (2013).

21. U. A. Gamm et al., "Measurement of tissue scattering properties using multi-diameter single fiber reflectance spectroscopy: in silico sensitivity analysis," Biomed. Opt. Express 2(11), 3150-3166 (2011).

22. A. Amelink, D. J. Robinson, and H. J. C. M. Sterenborg, "Confidence intervals on fit parameters derived from optical reflectance spectroscopy measurements," J. Biomed. Opt. 13(5), 054044 (2008).

23. W. H. Kruskal and W. A. Wallis, "Use of ranks in one-criterion variance analysis," Source J. Am. Stat. Assoc. 47(260), 583-621 (1952).

24. F. Miura et al., "Diagnosis of pancreatic cancer," HPB (Oxford) 8 , 337-342 (2006).

25. M. Erkan et al., "Cancer-stellate cell interactions perpetuate the hypoxia-fibrosis cycle in pancreatic ductal adenocarcinoma," Neoplasia 11(5), 497-508 (2009).

26. C. Feig et al., "The pancreas cancer microenvironment," Clin. Cancer Res. 18(16), 4266-4276 (2012).

27. D. Lytras et al., "Microvessel landscape assessment in pancreatic ductal adenocarcinoma: unclear value of targeting endoglin (CD105) as prognostic factor of clinical outcome,' Pancreas 44(1), 87-92 (2015).

28. L. R. McKenna and B. H. Edil, "Update on pancreatic neuroendocrine tumors," Gland Surg 3(4), 258-275 (2014).

29. J. Im and N. Rajaram, "Optical molecular imaging and spectroscopy of oxygenation and metabolism in tumors," IEEE J. Sel. Top. Quantum Electron. 22(3), 78-87 (2016).

30. M. Gerling et al., "Real-time assessment of tissue hypoxia in vivo with combined photoacoustics and high-frequency ultrasound," Theranostics 4(6), 604-613 (2014).

31. A. C. Koong et al., "Pancreatic tumors show high levels of hypoxia," Int. J. Radiat. Oncol. Biol. Phys. 48(4), 919-922 (2000).

32. N. C. Dhani et al., "Analysis of the intra- and intertumoral heterogeneity of hypoxia in pancreatic cancer patients receiving the nitroimidazole tracer pimonidazole," Br. J. Cancer 113(6), 864-871 (2015).

33. I. Lohse et al., "Assessment of hypoxia in the stroma of patient-derived pancreatic tumor xenografts," Cancers (Basel) 6(1), 459-471 (2014).

34. M. Erkan, M. Kurtoglu, and J. Kleeff, "The role of hypoxia in pancreatic cancer: a potential therapeutic target?" Expert Rev. Gastroenterol. Hepatol. 10(3), 301-316 (2015).

35. Q. Chang et al., "Hypoxia predicts aggressive growth and spontaneous metastasis formation from orthotopically grown primary xenografts of human pancreatic cancer," Cancer Res. 71(8), 3110-3120 (2011).
36. W. R. Wilson and M. P. Hay, "Targeting hypoxia in cancer therapy," Nat. Rev. Cancer 11(6), 393-410 (2011).

37. M. M. Alam et al., "A holistic view of cancer bioenergetics: mitochondrial function and respiration play fundamental roles in the development and progression of diverse tumors," Clin. Transl. Med. 5(3), 1-14 (2016).

38. T. Jansen and A. Daiber, "Direct antioxidant properties of bilirubin and biliverdin. Is there a role for biliverdin reductase?" Front. Pharmacol. 3(30), 1-10 (2012).

39. S. C. Kanick et al., "Method to quantitate absorption coefficients from single fiber reflectance spectra without knowledge of the scattering properties," Opt. Lett. 36(15), 2791-2793 (2011).

40. M. Yang et al., "Dual-color fluorescence imaging distinguishes tumor cells from induced host angiogenic vessels and stromal cells," Proc. Natl. Acad. Sci. U. S. A. 100(24), 14259-14262 (2011).

41. A. Suetsugu et al., "Multi-color palette of fluorescent proteins for imaging the tumor microenvironment of orthotopic tumorgraft mouse models of clinical pancreatic cancer specimens," J. Cell. Biochem. 113(7), 2290-2295 (2012).

42. H. J. M. Handgraaf et al., "Current and future intraoperative imaging strategies to increase radical resection rates in pancreatic cancer surgery," Biomed Res. Int. 2014, 1-8 (2014).

43. M. C. Boonstra et al., "Preclinical evaluation of a novel CEA-targeting near-infrared fluorescent tracer delineating colorectal and pancreatic tumors," Int. J. Cancer 137(8), 1910-1920 (2015).

44. J. W. Spliethoff et al., "Real-time in vivo tissue characterization with diffuse reflectance spectroscopy during transthoracic lung biopsy: a clinical feasibility study," Clin. Cancer Res. 22(2), 357-365 (2016).

Paulien L. Stegehuis received her bachelor's degree in technical medicine in 2009, and her master's degree in robotics and imaging in 2012, both at the University of Twente. Currently, he is a PhD student at the LUMC, partly at the image-guided surgery group, and partly at the Division of Image Processing. Her research focuses on the implementation of optical imaging techniques, such as optical coherence tomography and spectroscopy, to improve intraoperative tumor visualization.

Leonora S. F. Boogerd received her medical degree from Leiden University in 2013. Currently, he is a PhD student in the image-guided surgery group at the LUMC. Her research focuses on implementation of both nontargeted and targeted fluorescent probes during (oncologic) surgery. Furthermore, she is engaged with validation of imaging techniques, such as spectroscopy, to improve intraoperative tumor visualization.

Alexander L. Vahrmeijer is a surgical oncologist at the LUMC and head of the image-guided surgery group. His current research focuses on the introduction of image-guided surgery using newly developed imaging systems and clinically available probes and on the clinical introduction of probes and fluorophores.

Biographies for the other authors are not available. 